\title{
GENERALIZATION OF COLORING LINEAR TRANSFORMATION
}

\author{
Lukas NOVAK ${ }^{1}$, Miroslav VORECHOVSKY $Y^{1}$ \\ ${ }^{1}$ Institute of Structural Mechanics, Faculty of Civil Engineering, Brno University of Technology, \\ Veveri 331/95, Brno, Czech Republic \\ novak.1@fce.vutbr.cz, vorechovsky.m@vut.cz
}

DOI: $10.31490 /$ tces-2018-0013

\begin{abstract}
The paper is focused on the technique of linear transformation between correlated and uncorrelated Gaussian random vectors, which is more or less commonly used in the reliability analysis of structures. These linear transformations are frequently needed to transform uncorrelated random vectors into correlated vectors with a prescribed covariance matrix (coloring transformation), and also to perform an inverse (whitening) transformation, i.e. to decorrelate a random vector with a non-identity covariance matrix. Two well-known linear transformation techniques, namely Cholesky decomposition and eigendecomposition (also known as principal component analysis, or the orthogonal transformation of a covariance matrix), are shown to be special cases of the generalized linear transformation presented in the paper. The proposed generalized linear transformation is able to rotate the transformation randomly, which may be desired in order to remove unwanted directional bias. The conclusions presented herein may be useful for structural reliability analysis with correlated random variables or random fields.
\end{abstract}

\section{Keywords}

Linear transformation, correlation, Cholesky
decomposition, eigen-decomposition, structural
reliability, uncertainty quantification, random fields.

\section{Motivation}

Uncertainty quantification or probabilistic engineering analyses such as statistical, sensitivity or reliability analyses must often consider statistical dependencies among random variables. Whatever joint distribution function may be considered, the only analytically tractable random vector with correlated marginal distributions is multivariate Gaussian distribution, and the real problem with possibly non-Gaussian marginals is that they must often be transformed into Gaussian random vectors (we recall transformation methods such as Nataf or Rosenblatt transformation [1]).

An analyst using reliability methods must often make decisions performed with incomplete probability information. Indeed, in many practical cases, only the estimated correlation or covariance matrix is specified along with marginal distributions for individual random variables. In such a case, the information about the joint probability distribution function is not complete and one has to construct a density that fulfills this incomplete information. A solution is to have an underlying Gaussian random vector. This is a typical example where the normal-to-anything transformation (NORTA), sometimes known as the Nataf transformation, which is a special case of Rosenblatt transformation that considers the Gaussian copula, is used to construct the joint probability density of the random vector.

We also note that mathematical operations involving multivariate Gaussian random vectors are almost the only generally applicable tools currently available. Most of the methods from reliability engineering require two-way access from/to the transformed space of uncorrelated Gaussian random variables [2]. One can mention methods like Importance sampling, Asymptotic sampling, First Order Reliability Method (FORM) and Second Order Reliability Method (SORM), etc. Last but not least, most of the methods for the generation and analysis of random fields are based on linear transformations between uncorrelated and correlated Gaussian random vectors.

One of the simplest applications of linear transformation is the generation of realizations from a correlated random vector using Monte Carlo type simulation techniques, which is commonly used in practical applications of structural reliability analysis. The aim of this study is to review and compare available transformation techniques and the development of the generalized linear transformation technique. In the following section, the basic mathematical principle behind the linear transformation of Gaussian random vectors is presented. 


\section{Linear Transformation}

Let $\mathbf{Z} \sim \mathrm{N}(0, \mathbf{I})$ be the Gaussian random column vector for which the expected value $E\left[Z_{i} Z_{j}\right]=\delta_{i j}$, so the columns of $\mathbf{Z}$ are uncorrelated standard variables. We would like to transform $\mathbf{Z}$ into a Gaussian random vector $\mathbf{X} \sim \mathrm{N}(0, \boldsymbol{\Sigma})$, where $\boldsymbol{\Sigma}=\mathrm{E}\left[\mathbf{X X}^{\mathrm{T}}\right]$ is a target covariance matrix which is symmetric and positive definite.

A linear transformation maps from $n$-dimensional space to $m$-dimensional space. For simplicity, we shall start with a linear transformation between spaces of the same dimension:

$$
\mathbf{T}: \mathbf{R}^{n} \rightarrow \mathbf{R}^{n} .
$$

The linear transformation of $\mathbf{Z} \rightarrow \mathbf{X}$ (coloring) using a regular square transformation matrix $\mathbf{A}$ of order $n$ then reads:

$$
\mathbf{X}=\mathbf{A} \mathbf{Z} \text {. }
$$

The inverse transformation (whitening) is simply $\mathbf{Z}=\mathbf{A}^{-1} \mathbf{X}$. In order to establish the transformation, one has to determine a suitable transformation matrix $\mathbf{A}$ [3], based on the covariance matrix $\boldsymbol{\Sigma}$.

There are two well-known choices for $\mathbf{A}$ that will be reviewed next: a transformation based on the Cholesky decomposition of $\boldsymbol{\Sigma}$ or on the eigen-decomposition of $\boldsymbol{\Sigma}$.

The geometric properties of the transformation are given by matrix $\mathbf{A}$, as can be seen from Eq. (2). It is clear that both vectors can be rotated, stretched or flipped.

\section{Cholesky Decomposition}

The target covariance matrix can be decomposed using a lower triangular (Cholesky) matrix $\mathbf{L}$ as:

$$
\boldsymbol{\Sigma}=\mathbf{L} \mathbf{L}^{\mathrm{T}} \text {. }
$$

The coloring linear transformation with $\mathbf{A}_{\text {chol }} \equiv \mathbf{L}$ obtained by Cholesky $\boldsymbol{\Sigma}$ then reads:

$$
\mathbf{X}=\mathbf{A}_{\text {chol }} \mathbf{Z}=\mathbf{L Z} \text {. }
$$

This transformation guarantees that the uncorrelated vector Zgets transformed into $\mathbf{X}$ with the desired covariance matrix:

$$
\begin{aligned}
& \mathrm{E}\left[\mathbf{X} \mathbf{X}^{\mathrm{T}}\right]=\mathrm{E}\left[(\mathbf{L Z})(\mathbf{L Z})^{\mathrm{T}}\right]=\mathrm{E}\left[\mathbf{L} \cdot \mathbf{Z} \mathbf{Z}^{\mathrm{T}} \cdot \mathbf{L}^{\mathrm{T}}\right]= \\
& =\mathbf{L E}\left[\mathbf{Z} \mathbf{Z}^{\mathrm{T}}\right] \mathbf{L}^{\mathrm{T}}=\mathbf{L} \mathbf{L}^{\mathrm{T}}=\mathbf{\Sigma} .
\end{aligned}
$$

The backward transformation is obtained easily as

$$
\mathbf{Z}=\mathbf{L}^{-1} \mathbf{X} \text {. }
$$

In order to visualize the transformation, a numerical example is presented that involves two independent standard Gaussian random variables (vector $\mathbf{Z}$ ) that are transformed into standard Gaussian random vectors with covariance matrix $\boldsymbol{\Sigma}$ (in fact a correlation matrix because of standardized random variables). Given the target correlation matrix

$$
\boldsymbol{\Sigma}=\left[\begin{array}{ll}
1 & \rho \\
\rho & 1
\end{array}\right],
$$

the Cholesky matrix reads

$$
\mathbf{L}=\left[\begin{array}{cc}
1 & 0 \\
\rho & \sqrt{1-\rho^{2}}
\end{array}\right] .
$$

An explanatory illustration of the Cholesky linear transformation for the two-dimensional case is depicted in Fig. 1 for correlation coefficient $\rho=-0.6$. Note that the transformation matrix $\mathbf{L}$ is a lower triangular matrix with a unit on the first entry of the main diagonal, and therefore the first coordinate $x_{1}$ remains unchanged.

\section{Eigen-decomposition}

The decomposition of a symmetric positive definite matrix into eigenvectors and eigenvalues is usually called eigendecomposition. The method is applied under many different names, such as proper orthogonal decomposition, principal component analysis, orthogonal transformation of a covariance matrix, the main ingredient of the Karhunen-Loève expansion, etc. Any positive definite matrix can be decomposed as

$$
\boldsymbol{\Sigma}=\boldsymbol{\Phi} \lambda \boldsymbol{\Phi}^{\mathrm{T}}=\underbrace{\boldsymbol{\Phi} \lambda^{1 / 2}}_{\boldsymbol{A}_{\text {eig }}} \cdot \underbrace{\lambda^{1 / 2} \boldsymbol{\Phi}^{\mathrm{T}}}_{\boldsymbol{A}_{\text {eig }}^{\mathrm{T}}},
$$

where $\lambda$ is the diagonal matrix of (positive) eigenvalues of $\boldsymbol{\Sigma}$ (best if ordered from largest to smallest) and $\boldsymbol{\Phi}$ is the eigenvector matrix associated with the eigenvalues. Individual eigenvectors of $\boldsymbol{\Sigma}$ are orthogonal (recall the symmetry of $\boldsymbol{\Sigma}$ ) and they form columns of $\boldsymbol{\Phi}$. The coloring linear transformation with $\mathbf{A}_{\text {eig }}$ obtained by the eigen-decomposition of $\Sigma$ reads:

$$
\mathbf{X}=\mathbf{A}_{\mathrm{eig}} \mathbf{Z}=\left(\boldsymbol{\Phi} \boldsymbol{\lambda}^{1 / 2}\right) \mathbf{Z} .
$$

The backward transformation is obtained easily as

$$
\mathbf{Z}=\left(\boldsymbol{\Phi} \lambda^{1 / 2}\right)^{-1} \mathbf{X}=\boldsymbol{\lambda}^{-1 / 2} \boldsymbol{\Phi}^{\mathrm{T}} \mathbf{X}
$$

In this equation, the fact that the inverse of an orthogonal matrix is the transposed matrix $\boldsymbol{\Phi}^{-1}=\boldsymbol{\Phi}^{T}$ can be used. Also, the diagonal matrix $\lambda^{-1 / 2}$ has the inverse square roots of eigenvalues on the main diagonal. Having $\boldsymbol{\Phi}$ and $\boldsymbol{\lambda}$ at hand, the backward transformation is simple.

To show that transformation (10) yields the required covariance matrix, one can substitute for $\boldsymbol{\Sigma}$ as follows: 

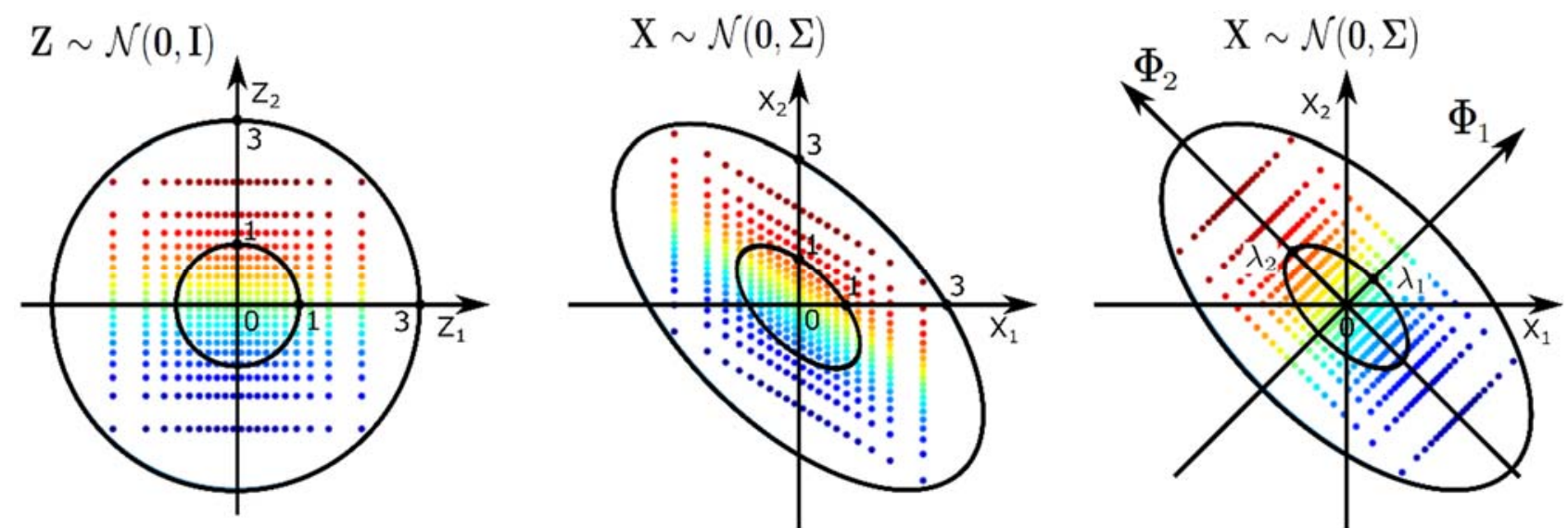

Fig. 1: Linear transformations of the original sample from $\mathbf{Z}$ (left), selected as an orthogonal grid of points. The sample of $\mathbf{X}$ is obtained from $\mathbf{Z}$ using: Cholesky decomposition (middle) and eigen-decomposition (right).

$$
\begin{aligned}
& \mathrm{E}\left[\mathbf{X} \mathbf{X}^{\mathrm{T}}\right]=\mathrm{E}[\underbrace{\boldsymbol{\Phi} \boldsymbol{\lambda}^{1 / 2} \mathbf{Z}}_{\mathbf{X}}(\underbrace{\boldsymbol{\Phi} \boldsymbol{\lambda}^{1 / 2} \mathbf{Z}}_{\mathbf{X}})^{\mathrm{T}}]= \\
& =\boldsymbol{\Phi} \boldsymbol{\lambda}^{1 / 2} \cdot \underbrace{\mathrm{E}\left[\mathbf{Z Z ^ { \mathrm { T } }}\right]}_{\mathrm{I}} \cdot \boldsymbol{\lambda}^{1 / 2} \boldsymbol{\Phi}^{\mathrm{T}}=\boldsymbol{\Phi} \boldsymbol{\lambda} \boldsymbol{\Phi}^{\mathrm{T}}=\boldsymbol{\Sigma} .
\end{aligned}
$$

eigen-decomposition has several advantages. First of all, the transformation of realizations to correlated space $\mathbf{X}$ keeps the original pattern from uncorrelated space $\mathbf{Z}$. This is illustrated using realizations of $2 \mathrm{D}$ vectors in Fig. 1 right. The solution to the eigenvalues and the associated orthonormal eigenvectors of the 2D correlation matrix in Eq. (7) is particularly simple:

$$
\lambda=\left[\begin{array}{cc}
1+\rho & 0 \\
0 & 1-\rho
\end{array}\right], \quad \boldsymbol{\Phi}=\left[\left(\begin{array}{l}
1 / \sqrt{2} \\
1 / \sqrt{2}
\end{array}\right)\left(\begin{array}{c}
-1 / \sqrt{2} \\
1 / \sqrt{2}
\end{array}\right)\right] .
$$

For computational purposes, a significant advantage of this method is the possibility of dimensional reduction using the transformation. The sum of eigenvalues is equal to the trace of $\boldsymbol{\Sigma}$ (denoted as $\operatorname{tr}(\boldsymbol{\Sigma})$ ), thus each eigenvalue represents information about variance carried by the corresponding eigenmode. If dimensionality reduction is required, it is possible to choose such eigenmode (variable) vectors associated with the largest eigenvalues that their sum forms a sufficient portion of $\operatorname{tr}(\boldsymbol{\Sigma})$ and the rest of the eigenmodes can be ignored. Usually, taking $0.95 \operatorname{tr}(\Sigma)$ suffices as it represents the $95 \%$ variability.

\section{Generalized Transformation}

Linear transformation can be generalized using eigenvalues and eigenvectors, which represent the principal directions - the axes of the ellipsoid. One can show that the desired covariance matrix can be decomposed as:

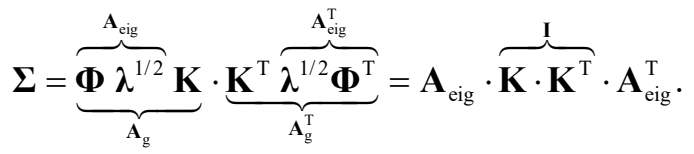

where $\mathbf{K}$ is an arbitrary orthogonal matrix. To show that such a transformation yields the required covariance matrix is simple:

$$
\begin{aligned}
& \mathrm{E}[\underbrace{\mathbf{A}_{\mathrm{g}} \mathbf{Z}}_{\mathbf{X}}(\underbrace{\mathbf{A}_{\mathrm{g}} \mathbf{Z}}_{\mathbf{X}})^{\mathrm{T}}]= \\
& =\boldsymbol{\Phi} \boldsymbol{\lambda}^{1 / 2} \mathbf{K} \underbrace{\mathrm{E}\left[\mathbf{Z} \mathbf{Z}^{\mathrm{T}}\right]}_{\mathbf{I}} \mathbf{K}^{\mathrm{T}} \boldsymbol{\lambda}^{1 / 2} \boldsymbol{\Phi}^{\mathrm{T}}=\boldsymbol{\Phi} \boldsymbol{\lambda} \boldsymbol{\Phi}^{\mathrm{T}}=\boldsymbol{\Sigma} .
\end{aligned}
$$

In other words, the generalized transformation matrix $\mathbf{A}_{\mathrm{g}}$ is obtained using $\mathbf{A}_{\text {eig }}$ by post-multiplication with an arbitrary orthogonal matrix and therefore establishes infinitely many possible transformation matrices between the correlated space $\mathbf{X}$ and the uncorrelated $\mathbf{Z}$-space:

$$
\mathbf{X}=\mathbf{A}_{\mathrm{g}} \mathbf{Z}=\left(\boldsymbol{\Phi} \boldsymbol{\lambda}^{1 / 2} \mathbf{K}\right) \mathbf{Z}
$$

The backward (whitening) transformation to uncorrelated space is then:

$$
\mathbf{Z}=\mathbf{A}_{\mathrm{g}}^{-1} \mathbf{X}=\left(\mathbf{K}^{\mathrm{T}} \boldsymbol{\lambda}^{-1 / 2} \boldsymbol{\Phi}^{\mathrm{T}}\right) \mathbf{X}
$$

The only difference between $\mathbf{A}_{\mathrm{g}}$ and $\mathbf{A}_{\text {eig }}$ lies in the post-multiplication by an orthogonal matrix which represents a unitary transformation (rotation). This rotational freedom can be extremely important in problems where the sample being transformed has a pattern that might interact with other features of the problem. The option of removing such an undesired pattern can help to eliminate bias that might lead statistical or reliability analyses to produce systematically erroneous results. Figure 2 displays an illustration of the generalization of linear transformation in $2 \mathrm{D}$, where random orthogonal matrices $\mathbf{K}$ are used.

The method for generating uniformly random orthogonal matrices can be found e.g. in [4]. As can be seen in Fig. 2, the choice of the orthogonal matrix $\mathbf{K}$ defines the position of the given realization on the same ellipsis. The ellipsis represents points at the same Mahalanobis distance. 

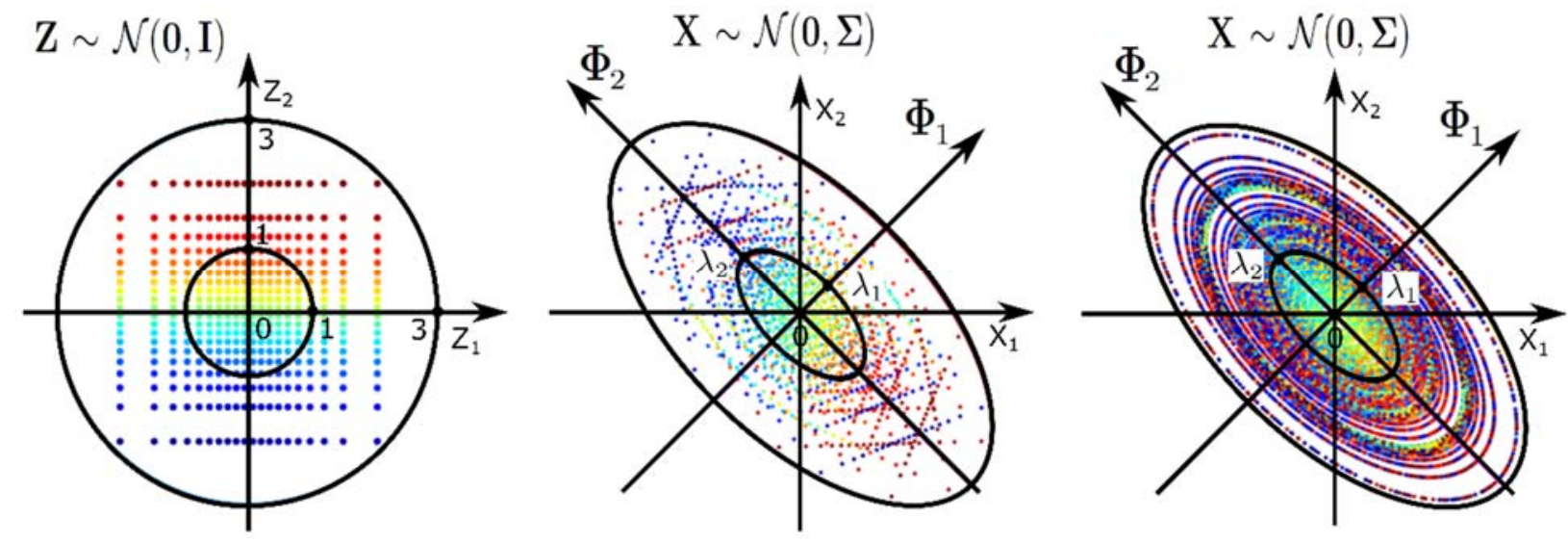

Fig. 2: Generalized linear transformations of the original sample from $\mathbf{Z}$ (left), selected as an orthogonal grid of points into various samples of $\mathbf{X}$ obtained using 4 random orthogonal matrices (middle) and 100 random orthogonal matrices (right). The realizations are at the same Mahalanobis distance.

The Mahalanobis distance $D$ is a multi-dimensional measure of the distance of a point from the mean value expressed as the number of standard deviations. In structural reliability analysis (e.g. the First Order Reliability Method) it is common to use reliability index $\beta$, which is defined as the shortest distance from the origin to the design point in uncorrelated standard normal space. Distance $D$ can be then viewed as the extension of $\beta$ to correlated normal space:

$$
\boldsymbol{\beta}=\sqrt{\mathbf{D}}=\sqrt{\mathbf{Z}^{\mathrm{T}} \mathbf{Z}}=\sqrt{(\mathbf{X}-\boldsymbol{\mu})^{\mathrm{T}} \mathbf{\Sigma}^{-1}(\mathbf{X}-\boldsymbol{\mu})} .
$$

A graphical interpretation of Mahalanobis distance in FORM is presented in Figure 3, where the original limit state function $f(\mathbf{X})$ is transformed to $\mathbf{Z}$ space by both methods. As can be seen, the position of the design point is different but the Mahalanobis distance is identical, which is crucial for reliability analysis. Note that $D$ is reduced to common Euclidean distance in $\mathbf{Z}$ space.

\section{Special Cases of Generalized Linear Transformation}

In this section, we will show that both commonly used transformation techniques, which specifically use either the Cholesky matrix or eigen-decomposition, can be viewed as special cases of the generalized transformation introduced in the previous section.

Since the choice of orthogonal matrix $\mathbf{K}$ is arbitrary, one can take it to be the identity matrix. Then, the generalized linear transformation becomes the eigendecomposition transformation.

Another possibility is to take the orthogonal matrix $\mathbf{K}=\mathbf{Q}$, where $\mathbf{Q}$ is the matrix from the $\mathrm{QR}$ decomposition [3]. Indeed, considering the transposed transformation matrix $\mathbf{A}_{\text {eig }}$, one can write:

$$
\mathbf{A}_{\text {eig }}^{\mathrm{T}}=\boldsymbol{\lambda}^{1 / 2} \boldsymbol{\Phi}^{\mathrm{T}}=\mathbf{Q R}
$$

Since $\mathbf{R}=\mathbf{L}^{\mathrm{T}}$ and the orthogonal matrix is selected as $\mathbf{K}=\mathbf{Q}$, the following equations show that the lower triangular matrix from Cholesky decomposition can be obtained using eigen-decomposition and $\mathbf{Q}$ :

$$
\begin{aligned}
& \boldsymbol{\Sigma}=\underbrace{\boldsymbol{\Phi} \boldsymbol{\lambda}^{1 / 2} \mathbf{Q}}_{\mathbf{A}_{\mathrm{g}}} \cdot \mathbf{Q}^{\mathrm{T}} \boldsymbol{\lambda}^{1 / 2} \boldsymbol{\Phi}^{\mathrm{T}}=\mathbf{L} \mathbf{L}^{\mathrm{T}}=\mathbf{L} \mathbf{R} \\
& \mathbf{L}=\underbrace{\boldsymbol{\Phi} \boldsymbol{\lambda}^{1 / 2} \mathbf{Q}}_{\mathbf{A}_{\mathrm{g}}} \cdot \mathbf{Q}^{\mathrm{T}} \boldsymbol{\lambda}^{1 / 2} \underbrace{\boldsymbol{\Phi}^{\mathrm{T}} \boldsymbol{\Phi}^{-\mathrm{T}}}_{\mathbf{I}} \boldsymbol{\lambda}^{-1 / 2} \mathbf{Q} \\
& \mathbf{L}=\boldsymbol{\Phi} \boldsymbol{\lambda}^{1 / 2} \mathbf{Q}
\end{aligned}
$$

\section{Conclusion}

In this paper, two well-known linear transformations of Gaussian random vectors are reviewed, and their relationships are clarified. The eigenvalue transformation is generalized using a random orthogonal matrix (rotation) in such a manner that it includes eigenvalue transformation and also Cholesky transformation as special cases. We remark that Cholesky transformation is computationally cheaper than eigenvalue transformation. The transformations find many applications in probabilistic modeling involving correlated random variables. One can name e.g. Nataf transformation to build a general nonGaussian joint distribution function, FORM and SORM, the generation of random fields and many others. The possibility of rotating the transformation randomly may be desired in order to remove unwanted directional bias of the eigenvalue transformation that might lead statistical or reliability analyses to produce systematically erroneous results. 

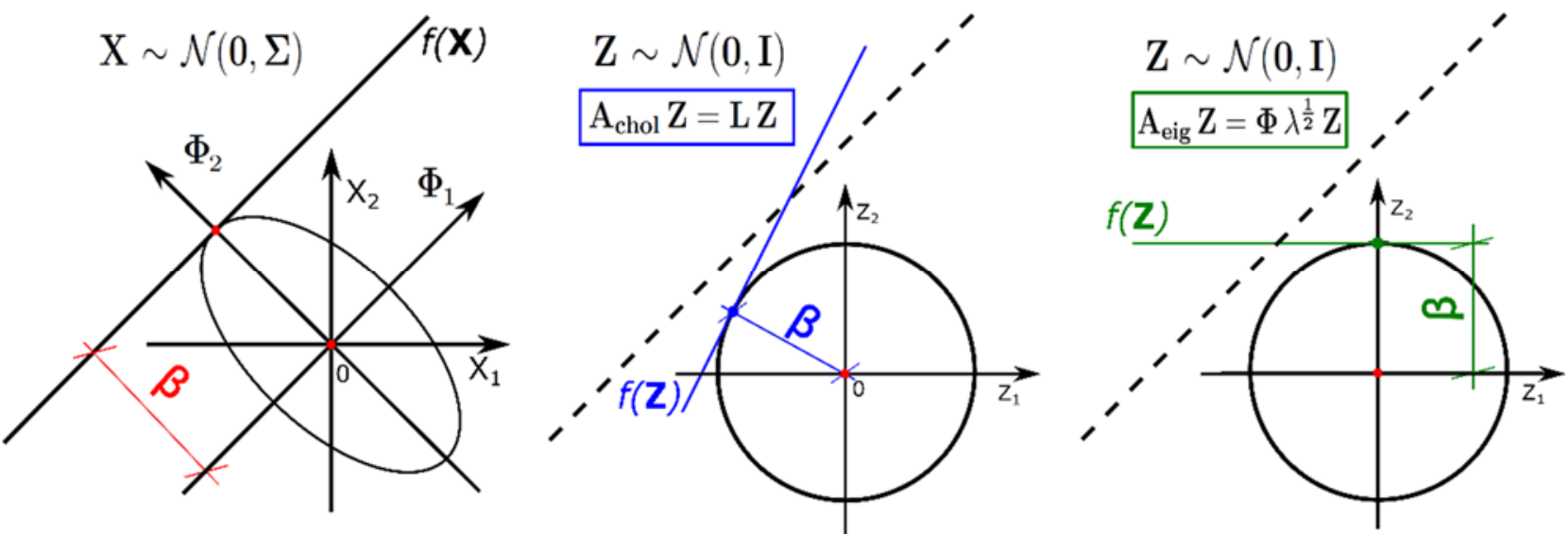

Fig. 3: The Mahalanobis distance in correlated (left) and uncorrelated space. The design point is transformed by Cholesky (middle) and eigen (right) decomposition-based transformation.

\section{Acknowledgments}

The authors give their thanks for the financial support of project No. LO1408 AdMaS UP - Advanced Materials, Structures and Technologies, awarded by the Ministry of Education of the Czech Republic under "National Sustainability Programme I".

\section{References}

[1] DER KIUREGHIAN, A.; LIU, P.-L. Structural Reliability under Incomplete Probability Information. Journal of Engineering Mechanics (ASCE), 1986, vol. 112, i. 1, doi 10.1061/(ASCE)0733-9399(1986)112:1(85), ISSN 0733-9399.

[2] VOŘECHOVSKÝ, M.; NOVÁK, D. Correlation control in small sample Monte Carlo type simulations I: A Simulated Annealing approach. Probabilistic Engineering Mechanics. Elsevier, 2009, vol. 24, i. 3, p. 452-462. ISSN 0266-8920.

[3] GENTLE, E. J. Computational statistics. New York: Springer Verlag, 2009. Statistics and Computing Series. ISBN 978-0-387-98143-7. 727 p.

[4] MEZZADRI FRANCESCO. How to generate random matrices from the classical compact groups. Notices of the American Mathematical Society, 2007, vol. 54, i. 5. p. 592604.

\section{About the Authors}

Lukáš NOVAK was born in Hustopece, Czech Republic. He received his M.Sc. from Brno University of Technology in 2018. His research interests include the reliability analysis of structures, specifically polynomial chaos expansion and semi-probabilistic methods.
Miroslav VORECHOVSKY was born in Brno, Czech Republic. He received his M.Sc. from Brno University of Technology in 2000 and his $\mathrm{PhD}$ in 2004. Since 2014 he has been engaged as a full professor at Brno University of Technology. His research interests include sampling methods, design of experiments, the reliability analysis of structures, random fields, extreme value statistics and the application of these probabilistic methods to the mechanics of concrete and other composites. 